V. V. Provotorov ${ }^{1}$, V. I. Ryazhskikh ${ }^{2}, Y u$. A. Gnilitskaya ${ }^{3}$

\title{
UNIQUE WEAK SOLVABILITY OF A NONLINEAR INITIAL BOUNDARY VALUE PROBLEM WITH DISTRIBUTED PARAMETERS IN A NETLIKE DOMAIN
}

1 Voronezh State University, 1, Universitetskaya square, Voronezh, 394006, Russian Federation

2 Voronezh State Technical University, 14, Moskovskii pr., Voronezh, 394026, Russian Federation

3 Air Force Academy named after professor N. E. Zhukovsky and Y. A. Gagarin, 54a, ul. Starikh Bol'shevikov, Voronezh, 396064, Russian Federation

This paper considers a fairly wide range of issues related to the solvability of the initial boundary value problem of the Navier-Stokes equations with distributed parameters on the net like region of the space $\mathcal{R}^{n}(n \geqslant 2)$. The authors here develop an idea, advanced in their work for the case of $n=1$ (the problems with distributed parameters on the graph), in the direction of the dimension increase $n$ and in forming the correct Hadamard conditions for the studied initial boundary value problem. The general scheme of the study is classical: the problem is solved in the functional space which is selected (the space of feasible solutions) and a special basis is formed for it, the problem of approximate solutions is settled by the Faedo-Galerkin method, for which a priori estimates of the energy inequalities type are set and the weak compactness of the family of these solutions is shown based on these estimates. Using nonburdensome conditions, the smoothness of the solution to the time variable is demonstrated. The uniqueness of the weak solution is shown in the particular case $n=2$, a feature quite often encountered in practice. The estimate for the norm of weak solution makes it possible to establish the continuous dependence of the weak solution from the initial data of the problem. The results obtained in this way are of interest to applications in the field of fluid mechanics and related sections of continuum mechanics, namely for the analysis of optimum control dynamics problems of multiphase media. It should be noted that the methods and approaches can be broadly generalized and are applicable to a wide class of nonlinear problems. Refs 20 .

Keywords: boundary value problem, distributed parameters on the netlike domain, the existence of a weak solution, the uniqueness conditions, Hadamard correctness.

\section{В. В. Провоторов ${ }^{1}$, В. И. Ряжских ${ }^{2}$ Ю. А. Гнилицкая}

\section{ОДНОЗНАЧНАЯ СЛАБАЯ РАЗРЕШИМОСТЬ НЕЛИНЕЙНОЙ НАЧАЛЬНО-КРАЕВОЙ ЗАДАЧИ С РАСПРЕДЕЛЕННЫМИ ПАРАМЕТРАМИ В СЕТЕПОДОБНОЙ ОБЛАСТИ}

1 Воронежский государственный университет, Российская Федерация, 394006, Воронеж, Университетская площадь, 1

Provotorov Vyacheslav Vasil'evich - doctor of physical and mathematical sciences, professor; wwprov@mail.ru

Ryazhskikh Viktor Ivanovich - doctor of engineering sciences, professor; ryazhskih_vi@mail.ru

Gnilitskaya Yuliya Aleksandrovna - PhD of physical and mathematical sciences, teacher; uliya al@mail.ru

Провоторов Вячеслав Василъевич - доктор физико-математических наук, профессор; wwprov@mail.ru

Ряжских Виктор Иванович - доктор технических наук, профессор; ryazhskih_vi@mail.ru

Гнилицкая Юлия Александровна - кандидат физико-математических наук, преподаватель; uliya_al@mail.ru

(C) Санкт-Петербургский государственный университет, 2017 
2 Воронежский государственный технический университет, Российская Федерация, 394026, Воронеж, Московский пр., 14

3 Военно-воздушная академия имени профессора Н. Е. Жуковского и Ю. А. Гагарина, Российская Федерация, 396064, Воронеж, ул. Старых большевиков, 54а

В работе рассматривается достаточно широкий круг вопросов, относящихся к разрешимости начально-краевой задачи типа Навье-Стокса с распределенными параметрами на сетеподобной области $\mathcal{R}^{n}(n \geqslant 2)$. Развиваются идеи, представленные в работах авторов для случая $n=1$ (задачи с распределенными параметрами на графе), в направлении увеличения размерности $n$ и формируются условия корректности по Адамару изучаемой начально-краевой задачи. Общая схема исследования остается классической: выбирается функциональное пространство, в котором решается задача (пространство допустимых решений) и формируется специальный базис для него, строятся приближения решений задачи по методу Фаэдо-Галеркина, для них устанавливаются априорные оценки типа энергетических неравенств и на основе этих оценок показывается слабая компактность построенного семейства решений. С помощью необременительных дополнительных условий находится гладкость решения по временной переменной. Единственность слабого решения рассматривается для частного случая $n=2$, достаточно часто встречающегося на практике. Оценка для нормы слабого решения дает возможность установить непрерывную зависимость слабого решения от исходных данных задачи. Результаты, полученные таким путем, представляют интерес для приложений в области гидромеханики и смежных разделах механики сплошных сред, а именно, для анализа задач оптимального управления динамикой многофазных сред. Следует отметить, что используемые методы и подходы обладают достаточно большой общностью и применимы к широким классам нелинейных задач. Библиогр. 20 назв.

Ключевые слова: начально-краевая задача, распределенные параметры на сетеподобной области, существование слабого решения, условия единственности, корректность по Адамару, слабые решения, оптимальное граничное управление, синтез управления, управляемость.

Introduction. This paper considers the existence of a weak solution of an $n$ dimensional nonlinear differential system with distributed parameters in a connected bounded netlike domain, which represents a geometric graph in the one-dimensional case. We introduce the space of admissible solutions and, using the Faedo-Galerkin method, establish the existence of a solution from the class of summable on a netlike domain functions. Taking into account the specifics of the Faedo-Galerkin method for the construction of approximate solutions in form of cut-off functions, we demonstrate that each such solution actually belongs to the space of functions with summable derivatives with respect to the time variable; the elements of this space satisfy an analog of the energy balance equation. The uniqueness of a weak solution is proved for the special case $n=2$. The obtained results can be used to analyze optimal control problems for differential distributed parameter systems in netlike domains, which have interesting analogies with multiphase problems of hydrodynamics. The paper continues the earlier research of [1-3].

Necessary notations, concepts and definitions. Consider an open bounded domain $\Im$ of the Euclidean space $\mathbb{R}^{n}(n \geqslant 2)$ that has a netlike structure [1], i. e. $\Im=\left(\bigcup_{k} \Im_{k}\right) \bigcup\left(\bigcup_{l} S_{l}\right)$, where $S_{l}$ is a surface that separates adjacent domains $\Im_{k}$, $\partial \Im$ indicates the boundary of $\Im$. The locus of conjugation of the adjacent domains $\Im_{k}$ will be called the node locus and further denoted by $\xi$; it represents the union of surfaces $S_{l}(\xi)$ whose number coincides with the number of conjugated domains, that is, $\xi=\bigcup_{l} S_{l}(\xi)$.

For a vector function $Y(x, t)=\left\{y_{1}(x, t), y_{2}(x, t), \ldots, y_{n}(x, t)\right\}\left(x=\left\{x_{1}, x_{2}, \ldots, x_{n}\right\}\right)$ defined in a domain $\Im_{T}=\Im \times(0, T)(T<\infty)$, consider the system 


$$
\begin{gathered}
\frac{\partial Y}{\partial t}-\nu \triangle Y+\sum_{i=1}^{n} Y_{i} \frac{\partial Y}{\partial x_{i}}=f-\operatorname{grad} p \\
\operatorname{div} Y=0\left(\sum_{i=1}^{n} \frac{\partial Y}{\partial x_{i}}=0\right)
\end{gathered}
$$

with the relationships

$$
\begin{gathered}
\left.Y\right|_{S_{l}^{-}(\xi)}=\left.Y\right|_{S_{l}^{+}(\xi)}, \\
\left.\sum_{l} \frac{\partial Y}{\partial n_{l}^{-}}\right|_{S_{l}^{-}(\xi)}+\left.\sum_{l} \frac{\partial Y}{\partial n_{l}^{+}}\right|_{S_{l}^{+}(\xi)}=0
\end{gathered}
$$

holding for each node locus $\xi$ (which are known in the literature as the conjugation conditions, see $[3,4])$; here $S_{l}^{-}(\xi)$ and $S_{l}^{+}(\xi)$ mean the unilateral surfaces for $S_{l}(\xi)$ defined by the direction of the normals $n_{l}^{-}$and $n_{l}^{+}$to the surfaces $S_{l}^{-}(\xi)$ and $S_{l}^{+}(\xi)$, respectively. Supplementing (1)-(4) with the initial conditions

$$
Y(x, 0)=Y_{0}(x), x \in \Im
$$

at time $t=0$ and the boundary conditions

$$
\left.Y\right|_{\partial \Im}=0
$$

we obtain the initial boundary value problem (1)-(6) to find the functions $Y(x, t)$ and $p(x, t)$ in the closed domain $\bar{\Im}_{T}=(\Im \cup \partial \Im) \times[0, T]$.

In the applied problems of hydrodynamics, the netlike domain $\Im$ is actually a hydrosystem that distributes the flows of a fluid (a multiphase medium); the function $Y(x, t)$ describes the velocity vector of the hydraulic flow in the domain $\Im_{T}$; relationships (1), (2) (the Navier-Stokes system in the evolutionary case [5, 6]) reflect the dynamics of an incompressible fluid with the coefficient of viscosity $\nu>0$ in the domain $\bigcup_{k} \Im_{k} \times(0, T)$; the balance equalities $(3)$, (4) define the fluid flow conditions in the node loci of the hydrosystem $\Im$.

Let us define a weak solution of the initial boundary value problem (1)-(6) (a turbulent solution in the terminology of [1]). To this end, introduce necessary spaces and present preliminary considerations.

Designate by $L_{2}(\Im)^{n}$ the space of measurable functions (classes) $\mu=\left\{\mu_{1}, \mu_{2}, \ldots, \mu_{n}\right\}$ that are square summable over the domain $\Im$. For $\mu, \rho \in L_{2}(\Im)^{n}$, the scalar product is defined by

$$
(\mu, \rho)=\sum_{i=1}^{n} \int_{\Im} \mu_{i}(x) \rho_{i}(x) d x,
$$

where $\|\mu\|=(\mu, \mu)^{1 / 2}$. Assume that $D(\Im)^{n}$ forms the space of functions that are infinitely differentiable in the domain $\Im$ and have compact supports in $\Im$.

Let $\mathfrak{D}(\Im)^{n}=\left\{\phi: \phi \in D(\Im)^{n}\right.$, $\left.\operatorname{div} \phi=0\right\}$ and denote by $\mathfrak{D}^{\prime}(\Im)^{n}$ the conjugate space for $\mathfrak{D}(\Im)^{n}$ (here and in the sequel, the symbol' indicates conjugate spaces). Define the space $\mathcal{H}(\Im)$ as the closure of $\mathfrak{D}(\Im)^{n}$ in the norm $L_{2}(\Im)^{n}$ with the scalar product $(7)$, $\|\mu\|_{\mathcal{H}(\Im)}=(\mu, \mu)^{1 / 2}$ and $\mathcal{H}(\Im)=\mathcal{H}(\Im)^{\prime}$.

Consider a space $\mathcal{H}^{1}(\Im)$ consisting of the elements $\mu \in L_{2}(\Im)^{n}$ that have the generalized derivative $\frac{\partial \mu}{\partial x} \in L_{2}(\Im)^{n}$, i. e. $\frac{\partial \mu}{\partial x_{i}} \in L_{2}(\Im), i=\overline{1, n}$. The space $\mathcal{H}^{1}(\Im)$ is equipped with the norm $\|\mu\|_{\mathcal{H}^{1}(\Im)}=\left(\|\mu\|_{L_{2}(\Im)}^{2}+\left\|\frac{\partial \mu}{\partial x}\right\|_{L_{2}(\Im)}^{2}\right)^{1 / 2}$ and represents a Hilbert space with the scalar product $(\mu, \rho)_{\mathcal{H}^{1}(\Im)}=(\mu, \rho)+\left(\frac{\partial \mu}{\partial x}, \frac{\partial \rho}{\partial x}\right)$. 
Next, define a space $V_{0}^{1}(\Im)$ as the closure in the norm $\mathcal{H}^{1}(\Im)$ for the set of elements $\mu \in \mathfrak{D}(\Im)^{n}$ satisfying the conjugation conditions

$$
\left.\sum_{l} \frac{\partial \mu}{\partial n_{l}^{-}(\xi)}\right|_{S_{l}^{-}(\xi)}+\left.\sum_{l} \frac{\partial \mu}{\partial n_{l}^{+}}\right|_{S_{l}^{+}(\xi)}=0 .
$$

In other words, $V_{0}^{1}(\Im)$ is the subspace of functions from $\mathcal{H}^{1}(\Im)$ that "satisfy the conjugation conditions" in all node loci $\xi$ of the domain $\Im$ and "vanish" on $\partial \Im$.

Consider the two forms

$$
\begin{gathered}
\rho(u, v)=\sum_{i, j=1}^{n} \int_{\Im} \frac{\partial u_{j}}{\partial x_{i}} \frac{\partial v_{j}}{\partial x_{i}} d x, \\
\varrho(u, v, \omega)=\sum_{i, k=1}^{n} \int_{\Im} u_{k} \frac{\partial v_{i}}{\partial x_{k}} \omega_{i} d x
\end{gathered}
$$

in functions $u, v, \omega$ such that the integrals in the representations above are convergent.

First, prove several auxiliary results that are similar to the ones in $[5$, p. $79-81 ; 6$, p. 71].

Lemma 1. The bilinear (8) and trilinear (9) forms are continuous on $V_{0}^{1}(\Im) \times V_{0}^{1}(\Im)$ and $L_{4}(\Im)^{n} \times V_{0}^{1}(\Im) \times L_{4}(\Im)^{n}$, respectively.

Proof. Applying the Cauchy-Bunyakowsky-Schwartz inequality to the functions $\frac{\partial u_{j}}{\partial x_{i}}$ and $\frac{\partial v_{j}}{\partial x_{i}}$ in the right-hand side of form (8) yields

$$
\left|\int_{\Im} \frac{\partial u_{j}}{\partial x_{i}} \frac{\partial v_{j}}{\partial x_{i}} d x\right| \leqslant \sqrt{\int_{\Im}\left(\frac{\partial u_{j}}{\partial x_{i}}\right)^{2} d x} \sqrt{\int_{\Im}\left(\frac{\partial v_{j}}{\partial x_{i}}\right)^{2} d x} \leqslant\left\|u_{j}\right\|_{V_{0}^{1}(\Im)}\left\|v_{j}\right\|_{V_{0}^{1}(\Im)} .
$$

By analogy, using the Cauchy-Bunyakowsky - Schwartz inequality in the right-hand side of form (9), first for the functions $u_{k} \omega_{i}$ and $\frac{\partial v_{i}}{\partial x_{k}}$ and then for the functions $u_{k}^{2}$ and $\omega_{i}^{2}$, we obtain

$$
\begin{gathered}
\left|\int_{\Im} u_{k} \frac{\partial v_{i}}{\partial x_{k}} \omega_{i} d x\right| \leqslant \sqrt{\int_{\Im}\left(u_{k} \omega_{i}\right)^{2} d x} \sqrt{\int_{\Im}\left(\frac{\partial v_{j}}{\partial x_{i}}\right)^{2} d x} \leqslant \\
\leqslant \sqrt[4]{\int_{\Im} u_{k}^{4} d x} \sqrt{\int_{\Im}\left(\frac{\partial v_{j}}{\partial x_{i}}\right)^{2} d x} \sqrt[4]{\int_{\Im} \omega_{i}^{4} d x} \leqslant\left\|u_{k}\right\|_{L_{4}(\Im)}\left\|v_{j}\right\|_{V_{0}^{1}(\Im)}\left\|\omega_{i}\right\|_{L_{4}(\Im)} .
\end{gathered}
$$

And the statement of this lemma follows from inequalities (10) and (11).

Corollary. Inequality (11) implies the continuity of form $(9)$ on $\left(V_{0}^{1}(\Im) \cap L_{4}(\Im)^{n}\right) \times$ $V_{0}^{1}(\Im) \times L_{4}(\Im)^{n}$.

Lemma 2. For any $u, \omega \in V_{0}^{1}(\Im)$, the following equalities are just:

1) $\varrho(u, u, \omega)=-\varrho(u, \omega, u)$;

2) $\varrho(u, \omega, \omega)=0$;

3) $\varrho(\omega, \omega, \omega)=0$.

Proof. The first statement of this lemma follows from representation (9) on integrating by parts the integrals in all terms over the domain $\Im$. Recall that the functions of the class $V_{0}^{1}(\Im)$ vanish on the boundary $\partial \Im$. And the second and third statements are a direct consequence of the first one.

Lemma 3. Let sequences $\left\{u_{m}\right\}_{m \geqslant 1}$ and $\left\{v_{m}\right\}_{m \geqslant 1}$ from $L_{2}(\Im)^{n}$ weakly converge in $L_{2}(\Im)^{n}$ to elements $u$ and $v$, respectively. Then the sequence $\left\{u_{m} v_{m}\right\}_{m \geqslant 1}$ has weak convergence in $L_{2}(\Im)^{n}$ to the element uv. 
Proof. It is necessary to show that

$$
\int_{\Im_{T}} u_{m} v_{m} \zeta d x d t \underset{m \rightarrow \infty}{\rightarrow} \int_{\Im_{T}} u v \zeta d x d t
$$

for any function $\zeta(x) \in \mathfrak{D}(\Im)^{n}$ satisfying the conjugation conditions at all node loci $\xi$ of the domain $\Im$ (or for any $\zeta(x) \in V_{0}^{1}(\Im)$, due to the density of the set of such functions in $\left.V_{0}^{1}(\Im)\right)$.

First, we take notice of an important fact ensuing from the weak convergence of the sequences $\left\{u_{m}\right\}_{m \geqslant 1}$ and $\left\{v_{m}\right\}_{m \geqslant 1}$ : the elements $u_{m}$ and $v_{m}$ are collectively bounded, i. e. $\left\|v_{m}\right\|_{L_{2}(\Im)^{n}}+\|v\|_{L_{2}(\Im)^{n}} \leqslant c$ and $\left\|u_{m}\right\|_{L_{2}(\Im)^{n}}+\|u\|_{L_{2}(\Im)^{n}} \leqslant c$. At the beginning, demonstrate that the sequence $\left\{v_{m} \zeta\right\}_{m \geqslant 1}$ strongly converges to $v \zeta$ in the space $L_{2}(\Im)^{n}$. Let $\epsilon>0$ be an arbitrarily small number. As $\zeta(x)$, it is possible to choose $\frac{\epsilon}{\|\zeta\|_{L_{2}(\Im) n}} \zeta(x)$; then the desired result follows from the chain of inequalities:

$$
\left\|v_{m} \zeta-v \zeta\right\|_{L_{2}(\Im)^{n}} \leqslant\left\|v_{m}-v\right\|_{L_{2}(\Im)^{n}}\|\zeta\|_{L_{2}(\Im)^{n}} \leqslant \epsilon\left(\left\|v_{m}\right\|_{L_{2}(\Im)^{n}}+\|v\|_{L_{2}(\Im)^{n}}\right) \leqslant \epsilon c .
$$

The statement of Lemma 3 is obvious from the estimates

$$
\begin{gathered}
\left|\int_{\Im} u_{m} v_{m} \zeta d x-\int_{\Im} u v \zeta d x\right|=\int_{\Im}\left|\left(u_{m} v_{m}-u v\right) \zeta\right| d x \leqslant \\
\leqslant \int_{\Im}\left(\left\|u_{m}\right\|_{L_{2}(\Im)^{n}}\left\|v_{m} \zeta-v \zeta\right\|_{L_{2}(\Im)^{n}}+\|v\|_{L_{2}(\Im)^{n}}\left\|u_{m} \zeta-u \zeta\right\|_{L_{2}(\Im)^{n}}\right) d x
\end{gathered}
$$

and the considerations above. The proof of Lemma 3 is finished.

Introduce the spaces of functions $u(x, t)$ of the variables $x, t \in \Im_{T}=\Im \times(0, T)$ $(T<\infty)$ and consider $u$ as a function of $t$ taking values in the space of functions of $x$. Particularly, if $V$ represents a Hilbert space, then denote by $L_{2}(0, T ; V)$ the space of functions (classes) $u:(0, T) \rightarrow V$ that are measurable, take values from $V$ and

$$
\|u\|_{L_{2}(0, T ; V)}=\left(\int_{0}^{T}\|u(t)\|_{V}^{2} d t\right)^{1 / 2}<\infty .
$$

Note that the equality $L_{2}\left(\Im_{T}\right)^{n}=L_{2}\left(0, T ; L_{2}(\Im)^{n}\right)$ holds naturally.

Next, introduce the following spaces: $W^{1,0}\left(\Im_{T}\right)$ as the space of functions $u(x, t) \in$ $L_{2}\left(\Im_{T}\right)^{n}$ that have the generalized first derivative with respect to $x$ belonging to $L_{2}\left(\Im_{T}\right)^{n}$, with the norm in $W^{1,0}\left(\Im_{T}\right)$ defined by

$$
\|u\|_{W^{1,0}\left(\Im_{T}\right)}=\left(\|u\|_{L_{2}\left(\Im_{T}\right)^{n}}^{2}+\left\|\frac{\partial u}{\partial x}\right\|_{L_{2}\left(\Im_{T}\right)^{n}}^{2}\right)^{1 / 2}
$$

$W^{1}\left(\Im_{T}\right)$ as the space of functions from $L_{2}\left(\Im_{T}\right)^{n}$ that have the generalized first derivatives also belonging to $L_{2}\left(\Im_{T}\right)^{n}$, with the norm defined by

$$
\|u\|_{W^{1}\left(\Im_{T}\right)}=\left(\|u\|_{L_{2}\left(\Im_{T}\right)^{n}}^{2}+\left\|\frac{\partial u}{\partial t}\right\|_{L_{2}\left(\Im_{T}\right)^{n}}^{2}+\left\|\frac{\partial u}{\partial x}\right\|_{L_{2}\left(\Im_{T}\right)^{n}}^{2}\right)^{1 / 2} .
$$

Using the properties of the traces of elements from $W^{1}\left(\Im_{T}\right)$ on each section of $\Im_{T}$ by the plane $t=t_{0}\left(t_{0} \in[0, T]\right)$ as elements of $L_{2}(\Im)^{n}$ that are continuous in $t$ in the norm $L_{2}(\Im)^{n}$ (see [7, p. 70]), define $\Omega_{0}\left(\Im_{T}\right)$ as the set of functions $u(x, t) \in W^{1}\left(\Im_{T}\right)$ belonging to the class $V_{0}^{1}(\Im)$ for fixed $t \in[0, T]$. Denote by $W_{0}^{1}\left(\Im_{T}\right)$ the closure of the set $\Omega_{0}\left(\Im_{T}\right)$ in the norm $W^{1}\left(\Im_{T}\right)$. 
Next, let $\widehat{\Omega}_{0}\left(\Im_{T}\right)$ be the set of all functions $u(x, t) \in W^{1,0}\left(\Im_{T}\right)$ that

a) have the finite norm

$$
\|u\|_{2, \Im_{T}}=\max _{0 \leqslant t \leqslant T}\|u(\cdot, t)\|_{L_{2}(\Im)^{n}}+\left\|\frac{\partial u}{\partial x}\right\|_{L_{2}\left(\Im_{T}\right)^{n}}
$$

b) have the trace defined on the sections of the domain $\Im_{T}$ by the plane $t=t_{0}$ $\left(t_{0} \in[0, T]\right)$ as a function of the class $V_{0}^{1}(\Im)$, i. e. for each element $u \in \widehat{\Omega}_{0}\left(\Im_{T}\right)$ and fixed $t \in[0, T]$, there exists a sequence $\left\{u_{n}\right\}$ of functions $u_{n}(x, t) \in V_{0}^{1}(\Im)$ converging to this trace in the norm $\mathcal{H}^{1}(\Im)$;

c) for each $t \in[0, T]$, belong to the space $V_{0}^{1}(\Im)$ and are continuous in $t$ in the norm $\mathcal{H}^{1}(\Im)$ on $[0, T]$, i. e. for any $t \in[0, T],\|u(\cdot, t+\Delta t)-u(\cdot, t)\|_{\mathcal{H}^{1}(\Im)} \rightarrow 0$ as $\Delta t \rightarrow 0$ uniformly on the interval $[0, T]$.

Designate by $V_{0}^{1,0}\left(\Im_{T}\right)$ the closure of the set $\widehat{\Omega}_{0}\left(\Im_{T}\right)$ in norm $(12)$; clearly, $V_{0}^{1,0}\left(\Im_{T}\right) \subset$ $W^{1,0}\left(\Im_{T}\right)$.

Remark 1. If $Y \in V_{0}^{1,0}\left(\Im_{T}\right)$, then $Y=0$ on $\partial \Im$, i. e. relationships (3), (4), and (6) should be treated as the conditions of belonging of $Y$ to the space $V_{0}^{1,0}\left(\Im_{T}\right)$. Equality (5) is considered almost everywhere on $\Im$.

Remark 2. The statements of Lemmas 1-3 remain in force for the functions that are defined in the domain $\Im_{\tau}=\Im \times(0, \tau)$ and have traces for any $t \in(0, \tau)$, where $\tau$ takes any fixed value within the interval $[0, T]$. The proofs of these results are exactly the same.

Remark 2 naturally leads to the following definition of a solution of problem (1)-(6), where the initial data (i. e. the functions $f$ and $Y_{0}$ ) satisfy the conditions

$$
f(x, t) \in L_{2,1}\left(\Im_{T}\right), \quad Y_{0}(x) \in \mathcal{H}(\Im) .
$$

Here $L_{2,1}\left(\Im_{T}\right)$ is a space whose elements belong to $L_{1}\left(\Im_{T}\right)$ and have the finite norm $\|f\|_{L_{2,1}\left(\Im_{T}\right)}=\int_{0}^{T}\left(\int_{\Im} f^{2} d x\right)^{1 / 2} d t, L_{2}\left(\Im_{T}\right) \subset L_{2,1}\left(\Im_{T}\right)$.

Definition. A weak solution of the initial boundary value problem (1)-(6) is a pair $\{Y, p\}$ as follows. The function $Y(x, t) \in V_{0}^{1,0}\left(\Im_{T}\right)$ satisfies the integral identity

$$
\begin{aligned}
(Y(x, t), \eta(x, t)) & -\int_{\Im_{t}} Y(x, \tau) \frac{\partial \eta(x, \tau)}{\partial \tau} d x d \tau+\nu \int_{0}^{t} \rho(Y, \eta) d \tau+\int_{0}^{t} \varrho(Y, Y, \eta) d \tau= \\
& =\left(Y_{0}(x), \eta(x, 0)\right)+\int_{\Im_{t}} f(x, \tau) \eta(x, \tau) d x d \tau
\end{aligned}
$$

for any $t \in[0, T]$ and any $\eta(x, t) \in W_{0}^{1}\left(\Im_{T}\right)$, while the function $p(x, t)$ belongs to the class $\mathfrak{D}^{\prime}\left(\Im_{T}\right)^{n}$. Here $\mathfrak{D}^{\prime}\left(\Im_{T}\right)^{n}$ is the conjugate space for the space $\mathfrak{D}\left(\Im_{T}\right)^{n}$, the elements of $\mathfrak{D}\left(\Im_{T}\right)^{n}$ are infinitely differentiable in $\Im_{T}$ functions with a compact support from $\Im_{T}$ ( see the analogous spaces $\mathfrak{D}(\Im)^{n}$ and $\left.\mathfrak{D}^{\prime}(\Im)^{n}\right)$.

Remark 3. Despite the apparent rigor, the above definition of a solution of problem (1)-(6) (i. e. a pair $\{Y, p\}$ ) has the explicit ambiguity caused by the variational statement (14) of this problem "eliminating" the function $p(x, t)$. Notably, there is no information concerning the function $p(x, t)$ except for relationship (14), and hence it suffices to find an appropriate function $p(x, t)$ within the class, i. e. $p(x, t) \in \mathfrak{D}^{\prime}\left(\Im_{T}\right)^{n}$. In terms of applications, this is an acceptable condition that guarantees the nonzero fluid dynamics in the domain $\Im_{T}$ (as a matter of fact, in many applications $p(x, t)$ is an a priori given function). According to the aforesaid, in the sequel we consider the function $Y(x, t)$ as a "solution" of problem (1)-(6); the existence of the function $p(x, t)$ and its belonging to the class $\mathfrak{D}^{\prime}\left(\Im_{T}\right)^{n}$ directly follow from the existence of $Y(x, t)$ within the class $V_{0}^{1,0}\left(\Im_{T}\right)$. 
The forthcoming sections are dedicated to the existence of a weak solution of the initial boundary value problem (1)-(6). Some considerations are similar to the ones presented in $[5$, p. 77$]$.

Existence of weak solution of problem (1)-(6). The idea to prove the existence of at least one weak solution of problem (1)-(6) remains the same as in [4, 8] for similar distributed parameter problems on a geometrical graph (network), but there is an essential difference that forms the main obstacle. In particular, the domain of variation of the spatial variable in problem (1)-(6) is the bounded domain $\Im$ of the Euclidean space $\mathbb{R}^{n}$, i. e. the spatial variable and the function $Y(x, t)$ are both vectors. This feature causes additional technical difficulties, mostly affecting the structure and properties of the spaces that are selected to describe the weak solutions of problem (1)-(6). The above-mentioned spaces lose many "good" properties intrinsic to the spaces of the functions of one variable. The nonlinearity of problem (1)-(6) has no small share in this process (the term $\sum_{i=1}^{n} Y_{i} \frac{\partial Y}{\partial x_{i}}$ in (1) defines the convection of an incompressible fluid [9, p. 10]).

Prior to proving the existence of a weak solution of the initial boundary value problem (1)-(6), in the domain $\Im$ consider the spectral problem

$$
-\nu \triangle U=\lambda U,\left.\quad U\right|_{\partial \Im}=0,
$$

by analogy to its counterpart on a graph $\Gamma$ [2]. This problem is to find the set of numbers $\lambda$ each associated with at least one nontrivial solution $U(x) \in V_{0}^{1}(\Im)$ satisfying the identity

$$
\nu((U, \eta))=\lambda(U, \eta)
$$

for any function $\eta(x) \in V_{0}^{1}(\Im)$; here $((\cdot, \cdot))$ denotes the scalar product of the form

$$
((U, \eta))=\sum_{i=1}^{n}\left(\frac{\partial U}{\partial x_{i}}, \frac{\partial \eta}{\partial x_{i}}\right)_{L_{2}(\Im)^{n}}
$$

This means that $U(x)$ is a generalized eigenfunction from the class $V_{0}^{1}(\Im)$ and $\lambda$ is the corresponding eigenvalue.

Proposition 1. The eigenvalues are real and have finite multiplicities, and they can be indexed in the ascending order of their magnitudes taking into account multiplicities, i. e. $\left\{\lambda_{i}\right\}_{i \geqslant 1}$; the generalized eigenfunctions are indexed accordingly, $\left\{U_{i}(x)\right\}_{i \geqslant 1}$.

Proposition 2. The system of the generalized eigenfunctions $\left\{U_{i}(x)\right\}_{i \geqslant 1}$ forms an orthogonal basis in the space $V_{0}^{1}(\Im)$ and in the space $L_{2}(\Im)^{n}$.

These results are established by the methods presented in [2].

Remark 4. These propositions remain in force for the spectral problem, where the boundary condition $\left.U\right|_{\partial \Im}=0$ is replaced by the more general one of the form

$$
\frac{\partial U}{\partial n}+\left.\sigma U\right|_{\partial \Im}=0
$$

(each domain $\Im_{l}$ has a specific constant $\sigma$, and $\frac{\partial U}{\partial n}$ designates the derivative along the inner normal inside the domain $\Im$ ). In this case, the generalized eigenfunction belongs to the space $V^{1}(\Im)$ (the definitions of the spaces $V^{1}(\Im)$ and $V_{0}^{1}(\Im)$ differ in the boundary conditions, $\left.V\right|_{\partial \Im}=0$ and the above-mentioned general boundary condition, used in the description of the set $\Omega$ ) and also satisfies the identity

$$
\nu((U, \eta))+\sum_{\zeta \in \partial \Im} \sigma U \eta=\lambda(U, \eta)
$$

for any function $\eta(x) \in W_{0}^{1}\left(S_{l}, \Im\right), \lambda$ is an eigenvalue. 
Theorem 1. There exists at least one weak solution of the initial boundary value problem (1)-(6) for arbitrary finite $T>0$.

$\mathrm{P}$ r o o f. Use the system of the eigenfunctions $\left\{U_{i}(x)\right\}_{i \geqslant 1}$ as a basis to represent the approximate solution $Y_{m}(x, t)$ as the cut-off function

$$
Y_{m}(x, t)=\sum_{i=1}^{m} g_{i m}(t) U_{i}(x)
$$

(the scalar functions $g_{i m}(t)$ are absolutely continuous on $[0, T]$ ) that satisfies the system

$$
\begin{gathered}
\left(\frac{\partial Y_{m}}{\partial t}, U_{i}\right)+\nu \rho\left(Y_{m}, U_{i}\right)+\varrho\left(Y_{m}, Y_{m}, U_{i}\right)=\left(f, U_{i}\right), \quad i=\overline{1, m}, \quad t \in[0, T], \\
Y_{m}(x, 0)=Y_{0 m}(x),
\end{gathered}
$$

where $Y_{0 m}(x)=\sum_{i=1}^{m} g_{i m}^{0} U_{i}(x)\left(g_{i m}^{0}=g_{i m}(0)\right) ; Y_{0 m}(x) \rightarrow Y_{0}(x)$ in the norm $\mathcal{H}(\Im)$.

System $(15),(16)$ is a system of differential equations in the functions $g_{i m}(t)$, which allows to find $Y_{m}$ for any $t \in[0, T]$. Let us demonstrate this by obtaining the a priori estimates of the norms of $Y_{m}$ in $V_{0}^{1,0}\left(\Im_{T}\right)$.

Multiplication of (15) by $g_{i m}(t)$ and summation over $i=\overline{1, m}$ yield

$$
\frac{1}{2} \frac{\partial}{\partial t}\left\|Y_{m}\right\|_{L_{2}(\Im)^{n}}^{2}+\nu \rho\left(Y_{m}, Y_{m}\right)=\left(f, Y_{m}\right)
$$

(recall that $\varrho\left(Y_{m}, Y_{m}, Y_{m}\right)=0$ by statement 3 of Lemma 2). In expression (17), the lefthand side is $\frac{1}{2} \frac{\partial}{\partial t}\left\|Y_{m}\right\|_{L_{2}(\Im)^{n}}^{2}+\nu\left\|\left(Y_{m}\right)_{x}\right\|_{L_{2}(\Im)^{n}}^{2}$ and the right-hand side obeys the estimate $\left(f, Y_{m}\right) \leqslant\|f\|_{L_{2}(\Im)^{n}}\left\|Y_{m}\right\|_{L_{2}(\Im)^{n}}$. In combination with (17), it follows that

$$
\frac{1}{2} \frac{\partial}{\partial t}\left\|Y_{m}\right\|_{L_{2}(\Im)^{n}}^{2}+\nu\left\|\left(Y_{m}\right)_{x}\right\|_{L_{2}(\Im)^{n}}^{2} \leqslant\|f\|_{L_{2}(\Im)^{n}}\left\|Y_{m}\right\|_{L_{2}(\Im)^{n}} .
$$

And integration over $t$ between 0 and $t$ gives the inequality

$$
\begin{gathered}
\frac{1}{2}\left\|Y_{m}\right\|_{L_{2}(\Im)^{n}}^{2}+\nu \int_{0}^{t}\left\|\left(Y_{m}\right)_{x}\right\|_{L_{2}\left(\Im_{t}\right)^{n}}^{2} d \tau \leqslant \\
\leqslant \frac{1}{2}\left\|Y_{m}(\cdot, 0)\right\|_{L_{2}(\Im)^{n}}^{2}+\|f\|_{L_{2,1}\left(\Im_{t}\right)}^{2} \max _{\tau \in[0, t]}\left\|Y_{m}(\cdot, \tau)\right\|_{L_{2}(\Im)^{n}}
\end{gathered}
$$

for arbitrary $t \in[0, T]$.

Introduce the notation $z(t)=\max _{\tau \in[0, t]}\left\|Y_{m}(\cdot, \tau)\right\|_{L_{2}(\Im)}$ and multiply both sides of inequality (18) by 2 to get

$$
z^{2}(t)+2 \nu\left\|\left(Y_{m}\right)_{x}\right\|_{L_{2}\left(\Im_{t}\right)}^{2} \leqslant\left\|Y_{m}(\cdot, 0)\right\|_{L_{2}(\Im)^{n}} z(t)+2\|f\|_{L_{2,1}\left(\Im_{t}\right)}^{2} z(t),
$$

as long as $\left\|Y_{m}(\cdot, 0)\right\|_{L_{2}(\Im)^{n}}^{2} \leqslant z(t)$. Then

$$
z^{2}(t) \leqslant J(t), \quad\left\|\left(Y_{m}\right)_{x}\right\|_{L_{2}\left(\Im_{t}\right)^{n}}^{2} \leqslant \frac{1}{2 \nu} J(t),
$$

with $J(t)=\left\|Y_{m}(\cdot, 0)\right\|_{L_{2}(\Im)^{n}} z(t)+2\|f\|_{L_{2,1}\left(\Im_{t}\right)^{n}}^{2} z(t)$. The last two inequalities lead to the estimate

$$
\begin{aligned}
& \left\|Y_{m}\right\|_{2, \Im_{t}}=z(t)+\left\|\left(Y_{m}\right)_{x}\right\|_{L_{2}\left(\Im_{t}\right)^{n}} \leqslant\left(1+\frac{1}{\sqrt{2 \nu}}\right) J^{1 / 2}(t) \leqslant \\
& \leqslant\left(1+\frac{1}{\sqrt{2 \nu}}\right)\left(\left\|Y_{m}(\cdot, 0)\right\|_{L_{2}(\Im)^{n}}+2\|f\|_{L_{2,1}\left(\Im_{t}\right)}\right)^{1 / 2}\left\|Y_{m}\right\|_{2, \Im_{t}}^{1 / 2}
\end{aligned}
$$

or, for any $t \in[0, T]$, 


$$
\left\|Y_{m}\right\|_{2, \Im_{t}} \leqslant\left(1+\frac{1}{\sqrt{2 \nu}}\right)^{2}\left(\left\|Y_{m}(\cdot, 0)\right\|_{L_{2}(\Im)^{n}}+2\|f\|_{L_{2,1}\left(\Im_{t}\right)}\right) .
$$

Taking into consideration formula (19), the expansion $Y_{0} m(x)=\sum_{i=1}^{m} g_{i m}^{0} U_{i}(x)$, as the convergence $Y_{0 m}(x) \rightarrow Y_{0}(x)$ in the norm $\mathcal{H}(\Im)$, we have $\left\|Y_{m}(\cdot, 0)\right\|_{L_{2}(\Im)^{n}} \leqslant c\left\|Y_{0}\right\|_{L_{2}(\Im)^{n}}$ (where $c>0$ is a constant independent of $m$ ) and, using (19), the estimate

$$
\begin{aligned}
\left\|Y_{m}\right\|_{2, \Im_{t}} & \leqslant\left(1+\frac{1}{\sqrt{2 \nu}}\right)^{2}\left(c\left\|Y_{0}\right\|_{L_{2}(\Im)^{n}}+2\|f\|_{L_{2,1}\left(\Im_{t}\right)}\right) \leqslant \\
& \leqslant C^{*}\left(\left\|Y_{0}\right\|_{L_{2}(\Im)^{n}}+2\|f\|_{L_{2,1}\left(\Im_{t}\right)}\right),
\end{aligned}
$$

where $C^{*}>0$ is a constant independent of $m$. The resulting estimate (20) pursues two goals, namely,

1) for any index $m$, the norms of the approximate solutions $Y_{m}(x, t)$ and their generalized derivatives $\frac{\partial Y_{m}(x, t)}{\partial x}$ in the space $\mathcal{H}(\Im)$ are bounded by a constant $C$ that is independent of $m$, i. e.

$$
\begin{gathered}
\left\|Y_{m}\right\|_{L_{2}(\Im)^{n}} \leqslant C, \\
\left\|\frac{\partial Y_{m}(\cdot, t)}{\partial x}\right\|_{L_{2}(\Im)^{n}} \leqslant C ;
\end{gathered}
$$

2) for any index $m$, the norms of the approximate solutions $Y_{m}(x, t)$ are estimated by the norms of the initial data $Y_{0}(x)$ and $f(x, t)$ of the initial boundary value problem (1)-(6).

With this in mind, employ the following well-known result for a sequence $\left\{Y_{m}\right\}_{m \geqslant 1}$ with the collectively bounded norms (21) of elements [7, p. 31]. In a sequence $\left\{Y_{m}\right\}_{m \geqslant 1}$, it is possible to separate a subsequence $\left\{Y_{m_{k}}\right\}_{k \geqslant 1}$ that weakly converges in norm (12) to a certain element $Y \in V_{0}^{1,0}\left(\Im_{T}\right)$ (in fact, $\left\{Y_{m_{k}}\right\}_{k \geqslant 1}$ weakly converges to $Y$ in the norm $L_{2}\left(\Im_{T}\right)^{n}$ together with $\left.\frac{\partial Y_{m_{k}}}{\partial x}\right)$. Show that the element $Y(x, t)$ is a solution of problem (1)-(6).

Multiply expression (15) by an absolutely continuous on $[0, T]$ function $d_{i}(t)$, perform summation over $i=\overline{1, m}$ and integrate the result over $t$ between 0 and $t$ :

$$
\begin{aligned}
& \left(Y_{m}(x, t), \Phi_{m}(x, t)\right)-\int_{\Im_{t}} Y_{m}(x, \tau) \frac{\partial \Phi_{m}(x, \tau)}{\partial \tau} d x d \tau+\nu \int_{0}^{t} \rho\left(Y_{m}, \Phi_{m}\right) d \tau+ \\
& +\int_{0}^{t} \varrho\left(Y_{m}, Y_{m}, \Phi_{m}\right) d \tau=\left(Y_{0}(x), \Phi_{m}(x, 0)\right)+\int_{\Im_{t}} f(x, \tau) \Phi_{m}(x, \tau) d x d \tau,
\end{aligned}
$$

where $\Phi_{m}(x, t)=\sum_{i=1}^{m} d_{i}(t) U_{i}(x)$.

Denote by $\Sigma$ the set of all functions $\Phi_{m}(x, t)$ with arbitrary $d_{i}(t)$ possessing the above properties and with arbitrary natural indexes $m$. The set $\Sigma$ is dense in $W_{0}^{1}\left(\Im_{T}\right)$. This follows from the density of the set $\left\{U_{i}(x)\right\}_{i \geqslant 1}$ in $V_{0}^{1}(\Im)$, the continuity of $\Phi_{m}(x, t) \in \Sigma$ in $t \in[0, T]$, the belonging $\Phi_{m}(x, t) \in V_{0}^{1}(\Im)$ for each fixed $t \in[0, T]$, and the definition of the space $W_{0}^{1}\left(\Im_{T}\right)$.

In (22), fix the function $\Phi_{m}(x, t)=\Phi_{m^{*}}^{*}(x, t) \in \Sigma$, i. e.

$$
\Phi_{m^{*}}^{*}(x, t)=\sum_{i=1}^{m^{*}} d_{i}^{*}(t) U_{i}(x),
$$

and, starting from index $m_{k} \geqslant m^{*}$, pass to the limit in the above subsequence $\left\{Y_{m_{k}}\right\}_{k \geqslant 1}$. 
First of all, note that the integrals

$$
\int_{0}^{t} \rho\left(Y_{m_{k}}, \Phi_{m^{*}}^{*}\right) d \tau, \quad \int_{0}^{t} \varrho\left(Y_{m_{k}}, Y_{m_{k}}, \Phi_{m^{*}}^{*}\right) d \tau
$$

contain the terms mentioned in Lemma 3. According to the latter (also, see Remark 2), these integrals converge to

$$
\int_{0}^{t} \rho\left(Y, \Phi_{m^{*}}^{*}\right) d \tau, \quad \int_{0}^{t} \varrho\left(Y, Y, \Phi_{m^{*}}^{*}\right) d \tau
$$

respectively. Passage to the limit yields formula (22) for the limiting function $Y(x, t)$, and hence for $\eta(x, t)=\Phi_{m^{*}}^{*}(x, t)$ the function $Y(x, t)$ is a weak solution of the initial boundary value problem (1)-(6) from $V_{0}^{1,0}\left(\Im_{T}\right)$ due to the density of the set $\Sigma$ in $W_{0}^{1}\left(\Im_{T}\right)$.

To complete the proof, it remains to argue the existence of a function $p(x, t) \in$ $\mathfrak{D}^{\prime}\left(\Im_{T}\right)^{n}$. Here the line of reasoning is as follows. The obtained function $Y(x, t)$ as a weak solution of problem (1)-(6) satisfies identity (14) for $t=T$; therefore, by choosing formally $\frac{\partial Y}{\partial t}-\nu \triangle Y+\sum_{i=1}^{n} Y_{i} \frac{\partial Y}{\partial x_{i}}-f=F$, we have $(F, \eta)=0$ for any element $\eta \in \mathfrak{D}\left(\Im_{T}\right)^{n}$ due to (13) and (14) (recall that $\mathfrak{D}\left(\Im_{T}\right)^{n}$ is dense in $W_{0}^{1}\left(\Im_{T}\right)$, which means the belonging of $F$ to the space $\left.\mathfrak{D}^{\prime}\left(\Im_{T}\right)^{n}\right)$. Moreover, this element has the representation $F=-\operatorname{grad} p$, where the function $p(x, t)$ is a certain element of the space $\mathfrak{D}^{\prime}\left(\Im_{T}\right)^{n}$. The proof of Theorem 1 is finished.

Remark 5. The proof of this theorem contains a deeper statement regarding a weak solution $Y(x, t)$ : the function $Y(x, t)$ has the derivative $\frac{\partial Y(x, t)}{\partial t}$ with respect to the variable $t$ that belongs to the class $L_{2}(0, T)$, which follows from the representation of the elements $Y_{m_{k}}(x, t)$ of the subsequence $\left\{Y_{m_{k}}\right\}_{k \geqslant 1}$ for the limiting function $Y(x, t)$.

Unique weak solution of problem (1)-(6). The uniqueness of a weak solution of the initial boundary value problem (1) - (6) is a complicated issue due to the arbitrary dimension $n$ of the domain $\Im$. We manage to prove uniqueness in the special case $n=2$, which is widespread in applications (also see [5, p. 83]). Here a prerequisite is to slightly reduce the class $V_{0}^{1,0}\left(\Im_{T}\right)$ of weak solutions by defining a contraction $\widetilde{V}_{0}^{1,0}\left(\Im_{T}\right)$ as the closure of the set $\widetilde{\Omega}_{0}\left(\Im_{T}\right)$ in norm (12). The elements of the set $\widetilde{\Omega}_{0}\left(\Im_{T}\right)$ have the same properties as those of the set $\widehat{\Omega}_{0}\left(\Im_{T}\right)$ (see items a) - c)), except item c) as follows:

$\left.\mathrm{c}^{*}\right)$ for each $t \in[0, T]$, the elements belong to $V_{0}^{1}(\Im)$ and are absolutely continuous in $t$ in the norm $\mathcal{H}^{1}(\Im)$ on $[0, T]$.

In this case, $\widetilde{V}_{0}^{1,0}\left(\Im_{T}\right) \subset V_{0}^{1,0}\left(\Im_{T}\right)$, the functions $u(x, t)$ belong to $\widetilde{V}_{0}^{1,0}\left(\Im_{T}\right)$ and have the generalized derivative $\frac{\partial u(x, t)}{\partial t} \in L_{2}\left(0, T ; V_{0}^{1}(\Im)\right)$. Theorem 1 remains in force for the weak solutions of the class $\widetilde{V}_{0}^{1,0}\left(\Im_{T}\right)$, as its proof is the same as above; and the limiting function $Y(x, t)$ has the derivative with respect to $t$ in the class $L_{2}\left(0, T ; V_{0}^{1}(\Im)\right.$ ) (see Remark 5 to Theorem 1 ).

Prior to presenting the main result, let us prove the following lemma by analogy to its counterpart in [5, p. 83].

Lemma 4. If $n=2$, then there exists a constant $c(\Im)$ such that

$$
\|v\|_{L_{4}(\Im)^{2}} \leqslant c(\Im)\|v\|_{L_{2}(\Im)^{2}}^{1 / 2}\|v\|_{V_{0}^{1}(\Im)}^{1 / 2}
$$

for any $v \in V_{0}^{1}(\Im) \cap L_{4}(\Im)^{2}$.

Proof. First, show inequality (23) for any $v \in D(\Im)^{2}$. Extend the vector function 
$v(x)=\left(v_{1}(x), v_{2}(x)\right)$ to the whole space $\mathbb{R}^{2}$, defining it as 0 beyond the domain $\Im$. Introduce the notation $D_{i} v=\left\{\frac{\partial v_{1}(x)}{\partial x_{i}}, \frac{\partial v_{2}(x)}{\partial x_{i}}\right\}$ and majorize $v^{2}(x)$ in the equalities

$$
v^{2}(x)=2 \int_{-\infty}^{x_{i}} v\left(D_{i} v\right) d x_{i} \quad(i=1,2) .
$$

We have

$$
v^{2}(x) \leqslant 2 v_{1}\left(x_{2}\right), \quad v^{2}(x) \leqslant 2 v_{2}\left(x_{1}\right),
$$

where

$$
v_{1}\left(x_{2}\right)=\int_{-\infty}^{+\infty}|v|\left|D_{1} v\right| d x_{1}, \quad v_{2}\left(x_{1}\right)=\int_{-\infty}^{+\infty}|v|\left|D_{2} v\right| d x_{2},
$$

and the symbol $|\cdot|$ denotes the magnitude of a vector in $\mathbb{R}^{2}$. On this basis, the following chain of inequalities holds:

$$
\begin{gathered}
\|v\|_{L_{4}\left(\mathbb{R}^{2}\right)}^{4}=\int_{\mathbb{R}^{2}} v^{4}(x) d x=\int_{\mathbb{R}} \int_{\mathbb{R}} v^{4}(x) d x_{1} d x_{2} \leqslant 4 \int_{\mathbb{R}} v_{1}\left(x_{2}\right) d x_{2} \int_{\mathbb{R}} v_{2}\left(x_{1}\right) d x_{1}= \\
=4 \int_{\mathbb{R}^{2}}\left|v\left\|D_{1} v\left|d x \int_{\mathbb{R}^{2}}\right| v\right\| D_{2} v\right| d x \leqslant 4\|v\|_{L_{2}\left(\mathbb{R}^{2}\right)}\left\|D_{1} v\right\|_{L_{2}\left(\mathbb{R}^{2}\right)}\|v\|_{L_{2}\left(\mathbb{R}^{2}\right)}\left\|D_{2} v\right\|_{L_{2}\left(\mathbb{R}^{2}\right)}= \\
=4\|v\|_{L_{2}\left(\mathbb{R}^{2}\right)}^{2}\left\|D_{1} v\right\|_{L_{2}\left(\mathbb{R}^{2}\right)}\left\|D_{2} v\right\|_{L_{2}\left(\mathbb{R}^{2}\right)} \leqslant 2\|u\|_{L_{2}\left(\mathbb{R}^{2}\right)}^{2}\left(\left\|D_{1} v\right\|_{L_{2}\left(\mathbb{R}^{2}\right)}^{2}+\left\|D_{2} v\right\|_{L_{2}\left(\mathbb{R}^{2}\right)}^{2}\right),
\end{gathered}
$$

yielding the estimate

$$
\|v\|_{L_{4}\left(\mathbb{R}^{2}\right)} \leqslant \sqrt[4]{2}\|v\|_{L_{2}\left(\mathbb{R}^{2}\right)}^{1 / 2}\left(\sum_{i=1}^{2}\left\|D_{i} v\right\|_{L_{2}\left(\mathbb{R}^{2}\right)}^{2}\right)^{1 / 4}=\sqrt[4]{2}\|v\|_{L_{2}(\Im)}^{1 / 2}\left\|v_{x}\right\|_{L_{2}(\Im)}^{1 / 2}
$$

for any $v \in D(\Im)^{2}$. And this estimate gives inequality (23) for any $v \in V_{0}^{1}(\Im)$ due to the density of $D(\Im)^{2}$ in $V_{0}^{1}(\Im)$ with a fixed constant $c(\Im)>0$ that depends on the measure of the domain $\Im$ only. The proof of Lemma 4 is finished.

We will establish that the solution of the initial boundary value problem (1)-(6) is unique using estimate (23) in the following case, which is not very restricting in applications. Whenever required, the space $V_{0}^{1}(\Im)$ is replaced by the space $V_{0}^{1}(\Im) \cap L_{4}(\Im)^{2}$ but with the norm of $V_{0}^{1}(\Im)$. In this case, all statements above remain in force obviously.

Theorem 2. If the domain $\Im$ is of dimension 2, then the initial boundary value problem (1)-(6) has a unique weak solution in the space $\widetilde{V}_{0}^{1,0}\left(\Im_{T}\right)$.

Proof. Let $\left(Y_{1}, p_{1}\right)$ and $\left(Y_{2}, p_{2}\right)$ be two solutions of problem (1)-(6) that belong to the class $\widetilde{V}_{0}^{1,0}\left(\Im_{T}\right)$. In addition, denote $\omega=Y_{1}-Y_{2}$ and $p=p_{1}-p_{2}$. According to the definition of a weak solution $\omega$, we have the identity

$$
\begin{aligned}
& (\omega(x, t), \eta(x, t))-\int_{\Im_{t}} \omega(x, \tau) \frac{\partial \eta(x, \tau)}{\partial \tau} d x d \tau+\nu \int_{0}^{t} \rho(\omega, \eta) d \tau+ \\
& +\int_{0}^{t} \varrho\left(\omega, Y_{1}, \eta\right) d \tau+\int_{0}^{t} \varrho\left(Y_{1}, \omega, \eta\right) d \tau+\int_{0}^{t} \varrho(\omega, \omega, \eta) d \tau=0
\end{aligned}
$$

for any $t \in[0, T]$ and any $\eta(x, t) \in \widetilde{V}_{0}^{1,0}\left(\Im_{T}\right)$ (on the strength of $\widetilde{V}_{0}^{1,0}\left(\Im_{T}\right) \subset W_{0}^{1}\left(\Im_{T}\right)$ ). Since $\omega$ belongs to the class $\widetilde{V}_{0}^{1,0}\left(\Im_{T}\right)$, identity $(24)$ takes the form

$$
\begin{gathered}
\int_{\Im_{t}} \frac{\partial \omega(x, \tau)}{\partial \tau} \eta(x, \tau) d x d \tau+\nu \int_{0}^{t} \rho(\omega, \eta) d \tau+ \\
+\int_{0}^{t} \varrho\left(\omega, Y_{1}, \eta\right) d \tau+\int_{0}^{t} \varrho\left(Y_{1}, \omega, \eta\right) d \tau+\int_{0}^{t} \varrho(\omega, \omega, \eta) d \tau=0
\end{gathered}
$$

for any $t \in[0, T]$ and any $\eta(x, t) \in \widetilde{V}_{0}^{1,0}\left(\Im_{T}\right)$. 
By letting $\eta(x, t)=\omega(x, t) \in \widetilde{V}_{0}^{1,0}\left(\Im_{T}\right)$ in $(25)$, we arrive at the relationship

$$
\begin{gathered}
\frac{1}{2}\|\omega(\cdot, t)\|_{L_{2}(\Im)}^{2}+\nu \int_{0}^{t} \rho(\omega, \omega) d \tau+ \\
+\int_{0}^{t} \varrho\left(\omega, Y_{1}, \omega\right) d \tau+\int_{0}^{t} \varrho\left(Y_{1}, \omega, \omega\right) d \tau+\int_{0}^{t} \varrho(\omega, \omega, \omega) d \tau=0
\end{gathered}
$$

(note that $\frac{1}{2} \frac{d}{d t}\|\omega(\cdot, t)\|_{L_{2}(\Im)^{2}}^{2}=\left(\frac{\partial \omega(x, t)}{\partial t}, \omega(x, t)\right)$. Taking into account representation (8) $\left(\rho(\omega, \omega)=\sum_{i, j=1}^{n} \int_{\Im} \frac{\partial \omega_{j}}{\partial x_{i}} \frac{\partial \omega_{j}}{\partial x_{i}} d x=\left\|\omega_{x}(\cdot, t)\right\|_{L_{2}(\Im)^{2}}^{2}\right)$ and the relationships of Lemma 2 $\left(\varrho\left(Y_{1}, \omega, \omega\right)=0\right.$ and $\left.\varrho(\omega, \omega, \omega)=0\right)$, we obtain

$$
\frac{1}{2}\|\omega(\cdot, t)\|_{L_{2}(\Im)^{2}}^{2}+\nu \int_{0}^{t}\left\|\omega_{x}(\cdot, \tau)\right\|_{L_{2}(\Im)^{2}}^{2} d \tau=-\int_{0}^{t} \varrho\left(\omega, Y_{1}, \omega\right) d \tau .
$$

Now, estimate the absolute value of the integral in the right-hand side of formula (26) using (9) and (11):

$$
\left|\int_{0}^{t} \varrho\left(\omega, Y_{1}, \omega\right) d \tau\right| \leqslant \alpha_{1} \int_{0}^{t}\|\omega(\cdot, \tau)\|_{L_{4}(\Im)^{2}}^{2}\left\|Y_{1}(\cdot, \tau)\right\|_{V_{0}^{1}(\Im)} d \tau
$$

(here $\alpha_{1}>0$ is a fixed constant). The last inequality, in combination with $\|v\|_{L_{4}(\Im)} \leqslant$ $\sqrt[4]{2}\|v\|_{L_{2}(\Im)}^{1 / 2}\left\|v_{x}\right\|_{L_{2}(\Im)}^{1 / 2}($ see the proof of Lemma 4$)$, yields the estimate

$$
\left|\int_{0}^{t} \varrho\left(\omega, Y_{1}, \omega\right) d \tau\right| \leqslant \alpha_{2} \int_{0}^{t}\|\omega(\cdot, \tau)\|_{L_{2}(\Im)^{2}}\left\|\omega_{x}(\cdot, \tau)\right\|_{L_{2}(\Im)^{2}}\left\|Y_{1}(\cdot, \tau)\right\|_{V_{0}^{1}(\Im)} d \tau
$$

( $\alpha_{2}>0$ is a fixed constant). This inequality can be transformed into

$$
\left|\int_{0}^{t} \varrho\left(\omega, Y_{1}, \omega\right) d \tau\right| \leqslant \nu \int_{0}^{t}\left\|\omega_{x}(\cdot, \tau)\right\|_{L_{2}(\Im)}^{2} d \tau+\alpha_{3} \int_{0}^{t}\left\|Y_{1}(\cdot, \tau)\right\|_{V_{0}^{1}(\Im)}^{2}\|\omega(\cdot, \tau)\|_{L_{2}(\Im)^{2}}^{2} d \tau
$$

$\left(\alpha_{3}=\alpha_{2} /(4 \nu), \alpha_{2}\left\|\omega_{x}(\cdot, \tau)\right\|_{L_{2}(\Im)^{2}}\left\|Y_{1}(\cdot, \tau)\right\|_{V_{0}^{1}(\Im)}\|\omega(\cdot, \tau)\|_{L_{2}(\Im)^{2}} \leqslant \nu\left\|\omega_{x}(\cdot, \tau)\right\|_{L_{2}(\Im)^{2}}^{2}+\right.$ $\left.\alpha_{3}\left\|Y_{1}(\cdot, \tau)\right\|_{V_{0}^{1}(\Im)}^{2}\|\omega(\cdot, \tau)\|_{L_{2}(\Im)^{2}}^{2}\right)$. Using $(26)$, it appears that

$$
\|\omega(\cdot, t)\|_{L_{2}(\Im)^{2}}^{2} \leqslant 2 \alpha_{3} \int_{0}^{t}\left\|Y_{1}(\cdot, \tau)\right\|_{V_{0}^{1}(\Im)}^{2}\|\omega(\cdot, \tau)\|_{L_{2}(\Im)^{2}}^{2} d \tau .
$$

And the desired result $\omega(x, t)=0$ follows immediately, as $\|\omega(\cdot, 0)\|_{L_{2}(\Im)^{2}}^{2}=0$. The proof of Theorem 2 is finished.

Continuity in initial data. While proving Theorem 1, we have obtained the a priori estimates (20)

$$
\left\|Y_{m}\right\|_{2, \Im_{t}} \leqslant C^{*}\left(\left\|Y_{0}\right\|_{L_{2}(\Im)^{2}}+2\|f\|_{L_{2,1}\left(\Im_{t}\right)}\right)
$$

for any $t \in[0, T]$ and all elements of the sequence $\left\{Y_{m}\right\}_{m \geqslant 1}$, where $C^{*}$ is a constant that depends on the measure of $\Im$ only. Hence, these estimates hold for all elements of the subsequence $\left\{Y_{m_{k}}\right\}_{k \geqslant 1}$ converging to a weak solution $Y(x, t)$. Passage to the limit over the weakly convergent subsequence $\left\{Y_{m_{k}}\right\}_{k \geqslant 1}$ as $k \rightarrow \infty$ yields the estimate (at $t=T$ )

$$
\|Y\|_{2, \Im_{T}} \leqslant C^{*}\left(\left\|Y_{0}\right\|_{L_{2}(\Im)^{2}}+2\|f\|_{L_{2,1}\left(\Im_{T}\right)}\right) .
$$


Estimate (27) for the norm of a weak solution $Y(x, t)$ from the space $V_{0}^{1,0}\left(\Im_{T}\right)$ (which is also valid for a weak solution from the space $\left.\widetilde{V}_{0}^{1,0}\left(\Im_{T}\right)\right)$ brings to the following result.

Theorem 3. A weak solution of the initial boundary value problem (1)-(6) in the space $V_{0}^{1,0}\left(\Im_{T}\right)\left(\widetilde{V}_{0}^{1,0}\left(\Im_{T}\right)\right)$ continuously depends on the initial data $f(x, t)$ and $Y_{0}(x)$.

Conclusion. Note that all results derived here remain in force for $n=1$ (e. g. see [10, 11]). In this case, the netlike domain $\Im$ is a geometrical graph $\Gamma$, and relationships (3) and (4) at each internal node $\xi$ of this graph are transformed into the Kirchoff-type boundary conditions

$$
\sum_{\gamma \in R(\xi)} a(1)_{\gamma} \frac{\partial Y(1, t)_{\gamma}}{\partial x}=\sum_{\gamma \in r(\xi)} a(0)_{\gamma} \frac{\partial Y(0, t)_{\gamma}}{\partial x} .
$$

Here $Y: \Gamma_{T}=\Gamma \times(0, T) \rightarrow V_{0}^{1}\left(\Gamma_{T}\right)\left(V_{0}^{1}\left(\Gamma_{T}\right)\right.$ is an analog of the space $V_{0}^{1}\left(\Im_{T}\right)$ for the graph $\Gamma) ; Y_{\gamma}$ denotes the restriction of the function $Y$ to the edge $\gamma ; a(x)$ means a fixed measurable bounded on $\Gamma$ function that satisfies condition $(28) ; R(\xi)$ and $r(\xi)$ are the sets of edges directed to the node $\xi$ and from the node $\xi$, respectively. (All edges of the graph are parameterized by the interval $[0,1]$.) The described situation underlies the analysis of optimal control problems on networks $[4,8,12,13]$. The current paper further develops the ideas presented in $[1,3]$ for $n=1$ towards increasing the dimension $n$, as well as constructs the well-posedness conditions of the initial boundary value problem (1)-(6) in the sense of Hadamard. The general scheme is classical [5, 6], as it includes the following steps: forming a special basis for the spaces of admissible solutions; designing approximate solutions of the problem by the Faedo-Galerkin method; obtaining a priori estimates for the approximate solutions in terms of energy balance inequalities; finally, proving the compactness of the resulting family of solutions using the obtained estimates. The smoothness of the solution in the spatial variable is established by imposing additional requirements. The results yielded by this approach are of certain interest for applications in hydromechanics and allied branches of continuum mechanics, namely, for the analysis of optimal control problems for multiphase media dynamics. Interestingly, other researchers [14-18] considered alternative approaches to the analysis of control problems, yet with the same treatment of the admissible control existence conditions. Also note that the problem under study may have a stochastic component in the representation of equation (1), and in this case it is necessary to use the results established in [19, 20].

\section{References}

1. Gnilitskaya Yu. A. Matematicheskoe modelirovanie $i$ chislennoe issledovanie processov $v$ setepodobnyh ob'ektah, opisyvaemyh evoljucionnymi uravneniajmi [Mathematical modeling and numerical study of processes in netlike objects described by evolutionary equations]. Cand. Diss. of physical and mathematical sciences. Voronezh, Voronezh State Technical University, 2015, 224 p. (In Russian)

2. Volkova A. S., Provotorov V. V. Obobshhennye reshenija i obobshhennye sobstvennye funkcii kraevyh zadach na geometricheskom grafe [Generalized solutions and generalized eigenfunctions of boundary-value problems on a geometric graph]. Izvestija vysshih uchebnyh zavedenij. Matematika [Russian Mathematics. Proceeding of Higher Educatianal institutions], 2014, vol. 58, no. 3, pp. 1-13. (In Russian)

3. Volkova A. S., Gnilitskaya Yu. A., Provotorov V. V. On the solvability of boundary-value problems for parabolic and hyperbolic equations on geometrical graphs. Automation and Remote Control, 2014, vol. 75 , no. 2, pp. 405-412.

4. Provotorov V. V., Gnilitskaya Yu. A. Granichnoe upravlenie volnovoj sistemoj v prostranstve obobshhennyh reshenij na grafe [Boundary control of a wave system in the space of generalized solutions on a graph]. Vestnik of Saint Petersburg University. Series 10. Applied Mathematics. Computer Science. Control Processes, 2013, iss. 3, pp. 112-120. (In Russian)

5. Lions J.-L. Some methods of solving non-linear boundary value problems. Paris, Dunod-GauthierVillars, 1969. 581 p. (Russ. ed.: Lions J.-L. Nekotorye metody resheniya nelineinykh kraevykh zadach. Moscow, Mir Publ., 1972, 587 p.). 
6. Lions J.-L., Magenes E. Problemes aux limites non homogenes et applications [Nonhomogenes boundary value problems and supplement]. Paris, Dunod-Gauthier-Villars, 1968. 364 p. (Russ. ed.: Lions J.L., Magenes E. Neodnorodnye granichnye zadachi i ikh prilozheniya. Moscow, Mir Publ., 1971,371 p.).

7. Ladyzhenskaya O. A. Kraevye zadachi matematicheskoi fiziki [Boundary value problems of mathematical physics]. Moscow, Nauka Publ., 1973, 407 p. (In Russian)

8. Podvalny S. L., Provotorov V. V. Startovoe upravlenie parabolicheskoj sistemoj s raspredelennymi parametrami na grafe [Starting control of a parabolic system with distributed parameters on a graph]. Vestnik of Saint Petersburg University. Series 10. Applied Mathematics. Computer Science. Control Processes, 2015, iss. 3, pp. 126-142. (In Russian)

9. Kutateladze S. S., Styrikovich M. A. Gidrodinamkia gazozhidkostnykh sistem [Hydrodynamics of gas-liquid systems]. Moscow, Energiya Publ., 1976, 296 p. (In Russian)

10. Provotorov V. V. Boundary control of a parabolic system with distributed parameters on a graph in the class of summable functions. Automation and Remote Control, 2015, vol. 76, no. 2, pp. 318-322.

11. Podvalny S. L., Provotorov V. V. The questions of controllability of a parabolic systems with distributed parameters on the graph. Intern. Conference "Stability and Control Processes" in memory of V. I. Zubov $(S C P), 2015$, pp. 117-119.

12. Provotorov V. V. Optimal'noe upravlenie parabolicheskoj sistemoj s raspredelennymi parametrami na grafe [Optimal control of a parabolic system with distributed parameters on a graph]. Vestnik of Saint Petersburg University. Series 10. Applied Mathematics. Computer Science. Control Processes, 2014, iss. 3, pp. 154-163. (In Russian)

13. Potapov D. K. Optimal'noe upravlenie raspredelennymi sistemami jellipticheskogo tipa vysokogo porjadka so spektral'nym parametrom i razryvnoj nelinejnost'ju [Optimal control of higher order elliptic distributed systems with a spectral parameter and discontinuous nonlinearity]. Journal of Computer and System Sciences International, 2009, vol. 52, no. 2, pp. 180-185. (In Russian)

14. Aleksandrov A. Yu., Zhabko A. P. Ob ustojchivosti reshenij odnogo klassa nelinejnyh raznostnyh sistem [On stability of solutions to one class of nonlinear difference systems]. Sibirskij matematicheskij zhurnal [Siberian Mathematical Journal], 2003, vol. 44, no. 6, pp. 951-958. (In Russian)

15. Aleksandrov A., Aleksandrova E., Zhabko A. Asymptotic stability conditions for certain classes of mechanical systems with time delay. WSEAS Transactions on Systems and Control, 2014, vol. 9, pp. 388-397.

16. Aleksandrov A. Yu., Platonov A. V. Ob ustojchivosti i dissipativnosti nekotoryh klassov slozhnyh sistem [On stability and dissipativity of some classes of complex systems]. Avtomatika i telemehanika [Automation and Remote Control], 2009, vol. 70, no. 8, pp. 1265-1280. (In Russian)

17. Veremey E. I., Korchanov V. M. Mnogocelevaja stabilizacija dinamicheskih sistem odnogo klassa [Multiobjective stabilization of the dynamic systems from a certain class]. Avtomatika $i$ telemehanika [Automation and Remote Control], 1988, no. 9, pp. 126-137. (In Russian)

18. Veremey E. I., Sotnikova M. V. Stabilizacija plazmy na baze prognoza s ustojchivym linejnym priblizheniem [Plasma stabilization by prediction with stable linear approximation]. Vestnik of Saint Petersburg University. Series 10. Applied Mathematics. Computer Science. Control Processes, 2011, iss. 1, pp. 116-133. (In Russian)

19. Karelin V. V. Shtrafnye funkcii v zadache upravlenija processom nabljudenija [Penalty functions in the control problem of an observation process]. Vestnik of Saint Petersburg University. Series 10. Applied Mathematics. Computer Science. Control Processes, 2010, iss. 4, pp. 109-114. (In Russian)

20. Kamachkin A. M., Yevstafyeva V. V. Oscillations in a relay control system at an external disturbance. Control Applications of Optimization 2000: Proceedings of the 11th IFAC Workshop, 2000, vol. 2, pp. 459-462.

For citation: Provotorov V. V., Ryazhskikh V. I., Gnilitskaya Yu. A. Unique weak solvability of a nonlinear initial boundary value problem with distributed parameters in a netlike domain. Vestnik of Saint Petersburg University. Applied Mathematics. Computer Science. Control Processes, 2017, vol. 13, iss. 3, pp. 264-277. DOI: 10.21638/11701/spbu10.2017.304

Статья рекомендована к печати проф. А. П. Жабко.

Статья поступила в редакцию 17 января 2017 г.

Статья принята к печати 8 июня 2017 г. 